\title{
Les aspects juridiques de la régulation publique économique au Maroc
}

\author{
Ali Kairouani*
}

\section{Introduction}

La notion de« régulation » est apparue pour la première fois dans un texte législatif maro-

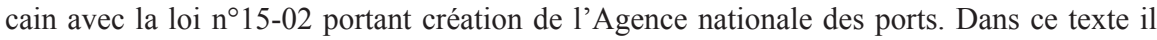
est spécifié que parmi les missions de cette agence existe « la régulation des activités et des opérateurs portuaires ».Toutefois, ce n'est pas la première autorité de régulation qui a vu le jour dans le système juridique marocain, puisqu'en 1993 et suite à la révision du Statut de Bank-al-Maghrib fut crée le Conseil déontologique des valeurs mobilières qui s'est transformé en Autorité marocaine du marché de capitaux afin de se moderniser et de se mettre en conformité avec la constitution de 2011 par le Dahir $n^{\circ} 1-13-21$ portant loi $n^{\circ} 43-12 .{ }^{1}$ On peut évoquer également l'agence nationale pour le développement des énergies renouvelables et de l'efficacité énergétique. Toutefois, il faut souligner qu'il existe deux catégories d'autorités de régulation économiques au Maroc. Celles que l'on vient de citer sont créées par des lois alors qu'il existe deux autorités de régulation économiques qui ont une origine constitutionnelle par le biais de l'article 166. Il s'agit du Conseil de la concurrence et de l'agence nationale de probité et de lutte contre la corruption. Dès lors, la fonction de régulation est d'une part, prévue par la constitution et d'autre part, consacrée par la loi. L'Etat régulateur ou l'émanation de l'Etat chargée de cette mission peuvent être définis comme « l'organe qui surveille et contrôle le secteur pour y maintenir l'équilibre entre le principe de concurrence et un autre principe». ${ }^{2}$ Cette rétrospective étymologique n'est pas le fruit du hasard mais explique avec une certaine mesure l'essence même de la régulation publique de l'économie marocaine. La prolifération des autorités administratives indépendantes dans les différents secteurs de l'économie nationale apporte une réponse à un besoin de s'adapter aux nouvelles exigences de l'économie de marché marocaine. Si l'on cite d'une part, par exemple la loi 20-13 créant le Conseil de la concurrence afin de veiller au respect du libre jeu de la concurrence dans le cadre de l'économie de marché, afin de garantir la compétitivité du tissu économique national et assurer un bon rapport qualité prix pour le bien être du consommateur. ${ }^{3}$ D'autre part, la nouvelle loi 60-16 portant création de l'agence marocaine

* Professeur assistant de Droit public à 1'Université Mohammed V de Rabat-Agdal, Maroc.

1 http://www.aderee.ma/index.php/fr/.

2 Marie-Anne FRISON-ROCHE, Les 100 mots de la régulation, Que-Sais-Je?, PUF, Paris 2011, p. 113.

3 Loi n²0-13 du 30 juin 2014 portant création du Conseil de la concurrence, Bulletin officiel, p. 3764. 
de développement des investissements et des exportations adoptée par le parlement marocain en août 2017 confirme ce choix fait par les pouvoirs publics afin de mettre en avant la régulation des flux d'investissement. L'Etat joue un double rôle dans l'économie marocaine puisqu'il est opérateur économique par le biais de ces entreprises publiques mais aussi garant de la libre et égale concurrence dans le marché marocain. Ce rôle de régulateur de l'Etat est incarné par l'intermédiaire de ses émanations de l'Etat à savoir les agences de régulation qui ont pour rôle de veiller au respect des règles de la concurrence de leur domaine de spécialité respective. Avec l'avènement de la nouvelle constitution il est apparu clairement que l'Etat avait cette intention d'ancrer définitivement cette fonction de régulateur. Il est impératif dès lors, d'analyser en premier lieu la nature normative de la régulation publique de l'économie au sein de l'ordre juridique marocain avant d'aborder en second lieu la question de la distinction à faire entre l'Etat régulateur et l'Etat actionnaire dans l'ordre juridique marocain. L'application accrue des règles internationales de concurrence à l'image des accords ratifiés par le Maroc dans le cadre de l'Organisation mondiale du commerce et des principes de libre concurrence remet directement en cause les modalités d'exercice de cette tutelle et ce, d'autant plus facilement qu'à l'instar des entreprises publiques qui en sont les destinataires, la tutelle de l'État ne fait pas l'objet, en droit administratif, d'un statut général. De même, le contrôle de l'État actionnaire sur l'entreprise publique, laquelle est conduite aujourd'hui à définir une stratégie de société commerciale pure et simple en raison du statut de société anonyme adoptée par plusieurs entreprises publiques marocaines. Il reste que, dans le silence de la loi, la tutelle administrative sur les entreprises publiques n'a toujours pas de statut constitutionnel propre après 2011. Il est alors facile au droit de la concurrence de redéfinir d'autres modes de relations entre l'Etat et les entreprises publiques, qui lui semblent plus conformes à la logique de marché : tel est le sens de la régulation. Ignorant délibérément la conception d'un Etat interventionniste dans la vie économique, l'existence même de l'Etat, le droit de la concurrence préconise aux pouvoirs publics ou à des institutions indépendantes, de définir un ensemble de mécanismes juridiques assortis le cas échéant de mécanismes extra-juridiques en vue d'établir ou de maintenir les grands équilibres économiques et de veiller à ce que l'intérêt général soit pris en compte. Ainsi, il sera question d'abord d'examiner les normes et les facteurs qui constituent l'essence et qui contribuent à l'ancrage de la régulation dans l'action publique au Maroc. Ensuite, la seconde partie de cette étude cherchera à analyser les lacunes de la régulation publique économique marocaine.

\section{La dimension normative de la régulation publique économique au Maroc}

Le développement de la régulation publique économique au Maroc a suivi un cheminement spécifique ce qui fera l'objet d'un examen de l'essence normative d'abord pour examiner ensuite les facteurs qui contribuent au recours intensif à la régulation publique économique. 


\subsection{L'essence normative de la régulation publique économique}

Avec la constitution marocaine de 2011 la régulation fait son entrée officielle au sein de l'ordre juridique marocain. Toutefois, il existe une essence juridique à la régulation publique de l'économique et une essence sociologique à celle-ci. Il est clair que le texte se réfère à cette notion à plusieurs reprises tout en maintenant les distinctions entre l'Etat régulateur à travers les autorités constitutionnelles chargées de réguler certaines activités économiques et l'Etat administration qui veille au maintien de la légalité et de la justice.Les lois qui ont créée les autorités administratives indépendantes sont des sources législatives de la régulation publique économique au Maroc. Elles ont contribuées depuis des années à l'introduction de cette notion dans le marché économique marocain. Les évolutions structurelles et conjoncturelles de l'économie au Maroc ont engendré l'évolution du mode de réglementation de cette dernière. La constitution marocaine de 2011 est la source fondamentale de cette régulation publique économique. Le texte est caractérisé par cette empreinte de régulation qui appartient à l'Etat qui l'exerce par le biais des autorités de régulation appelé dans le texte constitutionnel par instance de bonne gouvernance. Ces instances de bonne gouvernance et de régulationet à l'article 166 on Cite le conseil de la concurrence qui est l'autorité de régulation de la concurrence par excellence mais aussi à l'article 165 la Haute autorité de la communication et de l'audiovisuelle s'ajoute à cela l'autorité pour la probité cité dans l'article 167 et qui veille à instaurer une transparence et à mieux réguler les marchés publics tel que cela était prévu dans l'article 35 et l'article 36 pour le principe de la libre concurrence. Toutefois, il faut rappeler qu'il existe d'autres autorités de régulation économiques qui ont été créées par de simples textes législatifs et qui ne disposent pas du pouvoir de rendre des avis ou de trancher des différends relatifs aux activités économiques. L'élément commun entre toutes ses autorités de régulation économique demeure l'activité économique qui est l'objet de la régulation à savoir, le secteur audio qui a été libéralisé au Maroc contrairement au secteur visuel. Pour l'essence sociologique, elle réside dans la volonté de pouvoir gérer les entreprises publiques ainsi que réguler le marché économique en évitant les pratiques abusives qui existaient dans des pratiques administratives antérieures. Le constituant marocain a voulu établir un nouvel ordre économique national en instaurant la régulation concurrentielle comme clé de voûtede la nouvelle régulation de l'économie marocaine et autour d'elle des régulations satellitaires susceptibles de l'orienter. Les agences de régulation sont les satellites du conseil de la concurrence, il est certain que le caractère sectoriel de ces agences prouve la volonté de l'Etat de créer une distance entre ces fonctions exécutives et celles de réguler le marché. La norme constitutionnelle, ainsi que plusieurs normes législatives confortent la notion de régulation publique de l'économie au sein de l'ordre juridique marocain. Toutefois, l'influence du droit international ne peut être négligeable on peut citer à titre d'exemple les recommandations de l'OCDE particulièrement le rapport sur « l'Etat actionnaire, gouvernance des entreprises publiques » dont les travaux ont été organisés à Rabat le 14-15 septembre 2005. Cette influence internationale provient également des institutions financières internationales particulièrement la Banque 
Mondiale à travers l'indice Doing Business. Cet indice qui est relatif à la facilité de faire des affaires comprend plusieurs indicateurs relatif au droit en vigueur y compris la régulation publique de la concurrence qui devrait être assurée par les autorités publiques. ${ }^{4}$ Le Maroc occupe actuellement la $68^{\text {ème }}$ place au lieu de la $75^{\text {ème }}$ selon le rapport 2015 ce qui prouve qu'il a gagné 7 places en l'espace de deux ans. Ce changement est dû en partie aux différentes réformes législatives qui ont suivis l'avènement de la constitution de 2011 mais également à la réforme que connaît le système judiciaire marocain. ${ }^{5}$ Cette réforme a été confortée par la loi n³3-17 qui consacre l'indépendance du parquet au Maroc en transférant les pouvoirs de l'autorité gouvernementale au procureur du Roi auprès de la Cour de cassation qui a été promulguée le 25 septembre 2017. La mutation du système juridique marocain contribue amplement à son ouverture à la régulation mais reste très vulnérable face à une possible anomie générée essentiellement par l'inflation normative qui constitue une pathologie du système juridique. C'est pour cela qu'il serait important de s'intéresser aux facteurs qui contribue à l'émergence de la régulation publique économique après avoir rappelé les origines de celle-ci.

\subsection{Les facteurs d'émergence de la régulation publique économique}

L'existence du principe de la libre concurrence dans la constitution marocaine. Dès lors, l'Etat actionnaire est juge et partie, l'intérêt est de distinguer l'Etat actionnaire de l'Etat régulateur. L'Etat actionnaire est généralement un opérateur économique qui participe au jeu du marché mais sa seconde casquette est celle de l'Etat régulateur qui garantit la libre juste et égale concurrence entre tous les opérateurs économiques. A cet effet, il n'existe pour le moment aucune référence dans les textes à cet aspect même dans le code des bonnes pratiques de gouvernance des entreprises publiques qui a été adopté suite aux recommandations de l'Organisation de coopération et de développement économique sur le gouvernement d'entreprise Corporate gouvernance. Toutefois, la transparence est un élément sousjacent très important pour l'Etat actionnaire afin de d'accroitre la performance ce qui conduit à un lien direct avec le rôle de l'Etat régulateur. Il existe trois éléments constitutifs de la régulation publique économique. Primo, l'Etat se transforme d'un Etat tuteur à un Etat actionnaire, secundo, l'Etat régulateur supplante l'Etat interventionniste, tertio, l'Etat se transforme en entrepreneur afin de combler le vide dans certains secteurs stratégiques. Il faut rappeler néanmoins que ses mutations ne sont pas le fruit du hasard mais d'un long processus normatif. Il est certain que les crises conjoncturelles ou les transformations structurelles de l'Etat participe activement à l'évolution des techniques de gestion du patrimoine de l'Etat. Il semble très difficile d'établir une liste précise des instruments normatifs de la régulation publique toutefois certains aspects peuvent constituer un point de convergence

4 Rapport de la Banque Mondiale,Doing Business, 25 octobre 2016, p. 60.

5 Nadia BERNOUSSI, Mohammed MASLOUHI, « Les chantiers de la bonne justice. Contraintes et renouveau de la politique judiciaire au Maroc », Revue française de Droit constitutionnel, $\mathrm{n}^{\circ} 3$, 2013, p. 296. 
de ceux-là. L'Etat reste en tant que tel le principal régulateur de l'économie cela peut se faire par des moyens juridiques directs ou par des moyens juridiques indirects. D'un côté, il s'agit du gouvernement économique de l'Etat représenté principalement par le Ministère de l'Economie mais aussi par le Haut commissariat au plan qui est une instance constitutionnelle qui émet régulièrement des rapports à l'intention du gouvernement et du parlement. D'un autre côté, il peut s'agir des agences de régulation qui sont des émanations de l'Etat. Ce corpus normatif instauré pour la régulation publique de l'économie trouve son fondement juridique dans la constitution et dans les lois. L'une des principales lois qui participent activement à la régulation publique de l'économie directement ou indirectement demeure la loi de finances qui joue un rôle important dans l'investissement public par le biais du soutien financier à certaines entreprises publiques agissant dans des secteurs économiques stratégiques. La configuration normative au Maroc est très intéressante en raison de la rencontre entre des normes très différentes pour la régulation des activités économiques. Cependant, on distinguera uniquement ici les normes antérieures à la constitution marocaine de 2011 de tendance très libérale avec les normes postérieures à celle-ci. Pour les normes antérieures, on remarque l'absence de la notion de régulation au sein des normes régissant le marché économique ainsi que du rôle de l'Etat stratège dans le développement économique à travers les entreprises publiques. Concernant les normes postérieures, elles sont très libérales axées sur la libre concurrence, le contrôle et la transparence des activités économiques. Ce triptyque est composé par des facteurs de la régulation publique avec pour objectif d'établir un marché concurrentiel. L'une des difficultés de la réalisation de ce marché concurrentiel, c'est le statut obsolète de l'autorité de la concurrence qui est inactive depuis la réforme d'août 2014 date de la publication de la nouvelle loi 20-13 relative à la liberté des prix et de la concurrence. Les décisions de cette autorité de régulation de la concurrence même si pour certains juristes relèvent de la Soft law il n'en demeure pas moins qu'elles soient indispensables pour orienter les opérateurs économiques publics et privés vers de meilleurs pratiques. La volonté affichée d'étendre la libre concurrence aux marchés publics démontre le choix de l'ouverture. Cela constitue même une première dans l'ordre juridique marocain à savoir la rencontre entre les règles de la concurrence avec le droit administratif et les règles relatives à la commande publique. Cette rencontre est prévue par le décret n 2-12-349 du 20-03-2013 instaurant le nouveau code des marchés publics. L'article $1^{\text {er }}$ du décret dispose que parmi les règles auxquelles doit obéir la passation des marchés publics, existe la liberté d'accès à la commande publique; l'égalité de traitement des candidats; la transparence des procédures; la garantie des droits des concurrents; la moralisation de la passation et de l'exécution des marchés publics. ${ }^{6}$ Le constituant est allé plus loin en prévoyant une sanction dans l'article 36 de la constitution marocaine contre toute transgression de la part de l'administration des principes de passation des marchés publics dont le principe de la libre concurrence. Dès lors, ce principe de la libre concurrence contribue d'un côté, à la régulation à travers l'établissement d'une conduite 
respectueuse de la libre concurrence par l'administration et les opérateurs économiques. D'un autre côté, la conduite anticoncurrentielle conduit à une certaine sanction. Dans ce cadre, l'instance centrale de prévention de la corruption est chargée de mettre en œuvre un contrôle pour mettre en œuvre les dispositions de l'article 36 afin de lutter contre la corruption dans les marchés publics et de facto assurer une libre concurrence transparente. ${ }^{7}$

\section{Les lacunes normatives de la régulation publique économique au Maroc}

Les défaillances de la régulation publique économique au Maroc sont étroitement liées à l'absence d'une distinction formelle et substantielle entre certaines fonctions économiques de l'Etat.

\subsection{L'absence d'une distinction formelle des fonctions économiques de l'Etat}

Il est évident qu'il n'existe aucune distinction entre l'Etat régulateur et l'Etat actionnaire dans les textes juridiques au Maroc. En partant de la constitution qui est la norme suprême en passant par les lois et les règlements on ne rencontre aucune distinction. Toutefois, il existe dans le code des bonnes pratiques de gouvernance des entreprises et des Etablissements publics un renvoie à la notion d'actionnariat de l'Etat mais qui la confond volontairement ou involontairement avec la régulation. ${ }^{8}$ Pourtant dans le code de bonne gouvernance des entreprises publiques et même dans un rapport du Ministère de l'Economie de 2016 ainsi que dans le rapport de la Cour des comptes sur les Etablissements et les entreprises publiques en 2016 on emploi d'une manière récurrente l'expression Etat actionnaire. « L'usage de l'expression d' «État actionnaire » est à la fois un révélateur et sans doute un accélérateur de la banalisation de la gestion des entreprises publiques entraînée par l'application des règles de concurrence $" .^{9}$ Il est sûr que même dans le rapport de la Cour des comptes il semble qu'un flou subsiste concernant la distinction entre la régulation et l'actionnariat de l'Etat. La situation exceptionnelle de l'Agence nationale des ports qui est considérée comme un Etablissement public à caractère industriel et commercial alors que sa véritable fonction est d'être une autorité de régulation sectorielle. La société d'exploitation des ports est le véritable EPIC dans le domaine des ports. Tandis que l'ANP doit être élevée au rang de véritable autorité de régulation qui dispose d'un pouvoir de contrôle mais aussi de pouvoirs de polices. La distinction est à faire par les autorités compétentes et elle ne peut se réaliser qu'à partir du moment où l'Etat actionnaire verra fonctionnellement le jour à tra-

7 Projet de loi numéro 12-113, http://www.icpc.ma/wps/portal, octobre 2013.

8 Annexe du Code marocain de bonnes pratiques et de gouvernance des entreprises et Etablissements publics, p 6. « Réformes et libéralisations sectorielles pour conduire d'anciens monopoles publics à opérer dans un environnement concurrentiel, notamment, des opérateurs dans des secteurs stratégiques tels que les télécommunications, les infrastructures, le transport, l'énergie et l'audiovisuel ».

9 Martine LOMBARD, « Les conséquences juridiques du passage de l'Etat propriétaire à l'Etat actionnaire », Revue française de l'administration publique, 2007, n¹24, 575. 
vers une agence qui régulera les participations de l'Etat. Cette régulation du patrimoine de l'Etat permettra de prendre de la hauteur face à ce problème de confusion des compétences et surtout de gouvernance qui est inhérente à la configuration actuelle. La régulation publique des activités économiques sera assurée par le réseau d'agence ou d'autorités indépendantes à travers les différents secteurs. L'autorité de la concurrence quant à elle aura pour mission de coordonner ce réseau d'agence en rapport avec le droit de la concurrence. L'agence qui sera chargé du patrimoine de l'Etat sera tenue de respecter le principe de la libre concurrence dans le cadre des stratégies économiques menées sur le marché marocain. Cette agence des participations de l'Etat permettra de mieux organiser le patrimoine de l'Etat afin de mener une politique d'investissement plus cohérente et transparente. La position dominante qui appartient à plusieurs opérateurs historiques n'est pas en tant que telle interdite mais l'abus est strictement interdit par le droit de la concurrence. Le directeur actuel de l'autorité de la Concurrence remet en cause régulièrement les pratiques visant à encourager la rente au détriment de la libre, juste et égale concurrence au sein du marché économique marocain. ${ }^{10}$

\subsection{L'absence d'une distinction substantielle des fonctions économiques de l'Etat}

L'accent est mis généralement autour de l'Etat stratège et l'Etat actionnaire tout en oubliant les exigences de la nouvelle constitution à l'image de la régulation économique. Cette régulation de l'économie par l'Etat passe par sa garantie du principe de la libre concurrence. L'Etat est un opérateur économique à travers son rôle d'Etat actionnaire et de ce fait il est partie et juge au sein du marché économique. Le nouveau projet de loi relative à la gouvernance et au contrôle financier de l'Etat sur les Etablissements et autres entreprises publiques et autres organismes recèle plusieurs anomalies. ${ }^{11}$ L'article 3 du projet de loi recèle un véritable problème juridique puisqu'il dispose que « Le ministre chargé des finances exerce les droits et pouvoirs revenant à l'Etat en sa qualité d'actionnaire, au sein des assemblées d'actionnaires ». Toutefois, le ministre chargé des finances fait partie du gouvernement et pose les politiques publiques économiques donc il participe activement à la régulation positive ou négative du marché économique. Cette situation démontre clairement la confusion entre la fonction de régulation et la fonction d'actionnariat de l'Etat. Cela rappelle l'article déjà publié dans la REMALD en 2014 où il a été fait allusion à la création d'une agence indépendante pour la gestion des actifs de l'Etat et de son patrimoine. Le principe de la libre concurrence est battu en brèche par ce texte et n'est quasiment pas pris en considération. ${ }^{12}$ La Cour des comptes dans son rapport de 2016 reconfirme la même idée

10 Abdelali BENAMOUR, D'une société de rente à une société de mérite, La croisée des chemins, Casablanca, 2016, p. 256.

11 http://www.sgg.gov.ma/portals/0/AvantProjet/115/Avp_Loi_gouvernance_Fr.pdf..

12 Ali KAIROUANI, « La constitution économique du Maroc : étude analytique de droit public économique », REMALD, $\mathrm{N}^{\circ} 114,2014$, p 54. La dissociation entre l'Etat actionnaire et l'Etat régulateur trouve son fondement juridique dans le principe constitutionnel de la libre concurrence. Cette 
à la page 64 avec le titre suivant « fonction de l'Etat actionnaire non encore instaurée » et puis avec la recommandation qui va dans le sens de la proposition faite auparavant concernant la création d'une agence pour réguler le patrimoine de l'Etat. ${ }^{13}$ Il est évident qu'il existe une confusion dans l'esprit des décideurs publics autour de la notion de régulation et d'actionnariat puisqu'elles sont distinctes en raison du principe de libre concurrence. Ce principe constitutionnel contraint aujourd'hui les différents acteurs pour sa prise en considération, pour la mise en place d'un Etat régulateur et d'un Etat actionnaire distincts. Dès lors, il est clair que cette distinction formelle entre le rôle d'actionnaire de l'Etat et le rôle de régulateur de l'Etat passe indéniablement par le respect du principe de la libre concurrence et par la création d'une agence indépendante pour gérer le portefeuille de l'Etat. Cette autorité permettra d'administrer d'une manière transparente les actions de l'Etat dans le respect de la constitution. La présence d'une direction au sein du Ministère de l'économie qui gère le portefeuille de l'Etat participe à la confusion entre l'Etat stratège et l'Etat actionnaire en éliminant d'une manière factuelle l'Etat régulateur. Cette régulation économique est impérative pour la garantie d'une libre, juste et égale concurrence entre les opérateurs privés et publics. L'initiative privée ayant une place de plus en plus importante dans la vie économique du marché marocain, cette régulation publique est nécessaire afin de sauvegarder la compétitivité et la concurrence loyale. L'Etat régulateur a pour objectif de réaliser l'équilibre économique du marché à travers l'instauration d'un marché concurrentiel. Cette régulation est assurée principalement par les autorités de régulation qui sont des émanations de l'Etat et qui couvrent plusieurs secteurs d'activités économiques. Elles peuvent exister dans des secteurs de commerce visible ou de commerce invisible. Toutefois, le rôle de l'Etat actionnaire est à redéfinir ici puisqu'à aucun moment on évoque sa place dans ce système de régulation. Néanmoins, toute la portée de ces principes est encore assez incertaine. L'analyse normative du principe de séparation de l'opérateur et du régulateur reste encore floue quand il s'agit de séparer les fonctions de l'Etat actionnaire et de l'Etat régulateur. Le principe de séparation impose que l'autorité de régulation soit distincte de l'autorité qui assure les missions de l'Etat actionnaire. Cette absence de séparation claire entre l'Etat régulateur et l'Etat actionnaire porte atteinte au principe constitutionnel de la libre concurrence. Il existe un chevauchement entre la gouvernance et la régulation par l'Etat de ses participations au sein des entreprises publiques. La stratégie de l'Etat doit être calquée sur une gouvernance de ses participations au sein des entreprises publiques conformément au code des bonnes pratiques et de gouvernances des entreprises et des établissements publics. ${ }^{14}$ Les

liberté économique constitutionnalisée fait partie des Droits-libertés que l'Etat s'engage à garantir en tout état de cause. Dès lors, l'Etat opérateur économique devrait relever d'un autre organe que le ministère de l'économie afin de ne pas créer un imbroglio entre le pouvoir normatif et la liberté d'entreprendre également.

13 Rapport de la Cour des comptes du Royaume du Maroc de 2016 sur les Etablissements publics et les entreprises publique, p. 64.

14 Annexe du Code marocain de bonnes pratiques et de gouvernance des entreprises et Etablissements publics, pp. 11-16. 
conflits d'intérêts entre l'Etat actionnaire et l'Etat régulateur demeurent plausibles à l'heure actuelle.

\section{Conclusion}

Les obstacles à la régulation publique économique ne sont pas que d'une nature juridique mais ils peuvent avoir également un fondement méta-juridique. La matérialisation des obstacles principalement provient de l'environnement socioculturel qui refuse encore de s'inscrire parfaitement dans la cinétique normative que connait l'ordre juridique marocain. L'acculturation juridique est indispensable afin d'accompagner l'instauration de cette régulation publique de l'économie, par l'information ainsi que par l'amélioration du cadre institutionnel. La création d'une agence des participations de l'Etat permettrait à ce dernier de prendre de la distance par rapport à son rôle d'opérateur économique. L'action politique de l'Etat devrait s'inscrire dans une dimension plus impartiale et neutre à l'égard de ses activités économiques. Toutefois, la libre concurrence ne demeure pas qu'une affaire d'Etat mais elle concerne également les opérateurs privés qui continuent également à violer ouvertement les règles de la concurrence. En définitive, la corruption constitue une entrave pour une meilleure transparence dans la vie économique et une régulation performante de l'économie marocaine. L'instance nationale pour la probité et de lutte contre la corruption devrait jouer un rôle plus important afin de mieux purifier les activités économiques. 